\title{
LEVANTAMENTO E FLUTUAÇÃO POPULACIONAL DE PARASITOIDES DE MOSCAS- DAS-FRUTAS (DIPTERA: TEPHRITIDAE) DE OCORRÊNCIA EM GOIABEIRA (PSIDIUM GUAJAVA L.) EM FORTALEZA, CEARÁ
}

\author{
A.P. de Moura'; D.C.M. de Moura² \\ 1Embrapa Hortaliças, CP 218, CEP 70359-970, Brasília, DF, Brasil. E-mail: apmoura@cnph.embrapa.br
}

\section{RESUMO}

\begin{abstract}
Objetivou-se com o presente trabalho conhecer os himenópteros parasitoides associados às moscas-das-frutas (Diptera: Tephritidae) de ocorrência em pomar urbano de goiabeira (Psidium guajava L.) (Myrtaceae), em Fortaleza, Ceará, bem como determinar a flutuação populacional desses organismos nesse ecossistema. Realizaram-se coletas de frutos maduros em plantas de goiabeira e frutos recém-caídos, no período de novembro de 1999 a outubro de 2000. Os parasitoides obtidos pertencem às famílias Braconidae, Chalcididae, Figitidae (Eucoilinae), Diapriidae (Diapriinae) e Eulophidae, sendo que os da família Figitidae são os mais comuns. As maiores incidências desses organismos ocorreram nos meses de janeiro e março de 2000.
\end{abstract}

PALAVRAS-CHAVE: Anastrepha spp., Ceratitis capitata, Figitidae, Diapriidae, Hymenoptera.

\section{ABSTRACT}

\begin{abstract}
SURVEY AND POPULATIONAL DYNAMICSOF PARASITOIDSOF FRUIT FLIES(DIPTERA: TEPHRITIDAE) OCCURING IN GUAVA (PSIDIUM GUAJAVA L.) IN FORTALEZA, CEARÁ, BRAZIL. This study was aimed to identify the parasitoids associated to fruit-fly species (Diptera: Tephritidae) occurring in an urban guava (Psidium guajava L.) (Myrtaceae) orchard located in Fortaleza, Ceará State, Brazil, as well as to determine the populational dynamics of these organisms in the ecosystem. Ripe and newly fallen guava fruits, from November 1999 to December 2000 were collected. The parasitoids obtained belong to the families Braconidae, Chalcididae, Figitidae (Eucoilinae), Diapriidae (Diapriinae) and Eulophidae. The most common parasitoids belong to the family Figitidae. The highest occurrences were in January and March 2000.
\end{abstract}

KEY WORDS: Anastrepha spp., Ceratitis capitata, Figitidae, Diapriidae, Hymenoptera.

\section{INTRODUÇÃO}

A fruticultura no Nordeste brasileiro é uma atividade agrícola em pleno processo de expansão e que tem se destacado como segmento de grande importância socioeconômica. Dentre as diversas espécies frutíferas cultivadas nessa região, destacam-se a mangueira Mangifera indica L. (Anacardiaceae), a videira Vitis vinifera L. (Vitaceae), a bananeira Musa spp., (Musaceae) e a goiabeira Psidium guajava L. (Myrtaceae).

A goiabeira é originária das regiões tropicais americanas, ocorrendo de forma natural desde o México até o Sul do Brasil. Essa espécie é cultivada em todas as regiões tropicais e subtropicais do mundo (PEREIRA, 1995). O Brasil é o terceiro maior produtor mundial de goiaba, sendo os estados de
São Paulo, Pernambuco e Goiás, nesta ordem, os maiores produtores do país, que em conjunto respondem por cerca de $74 \%$ da produção nacional (Agrianual, 2009).

Apesar de sua relevância econômica no cenário mundial, a exportação da goiaba brasileira in natura ainda é inexpressiva, tornando sua comercialização dependente quase que exclusivamente do mercado interno (Costa et al., 2007). Uma das principais causas que justificam essa condição diz respeito à presença de pragas nas áreas cultivadas, notadamente de pragas quarentenárias, dentre elas as moscas-das-frutas (Diptera: Tephritidae), consideradas importantes pragas da fruticultura mundial, causando perdas significativas à produção e limitando o livre trânsito de frutas devido às restrições impostas por parte dos países importadores (MALAVASI, 2000).

${ }^{2}$ Universidade Federal Rural do Rio de Janeiro, Departamento de Engenharia, Instituto de Tecnologia, Seropédica, RJ, Brasil. 
As restrições à exportação dessa fruta, principalmente para países da União Europeia, que desde 2003 têm exigido frutos produzidos sob o sistema de produção integrada (KovALESKI; RIBEIRO, 2003), se devem, também, ao uso muitas vezes indiscriminado de agrotóxicos que apresentam elevada toxicidade, que deixam resíduos nos frutos, além de afetarem a entomofauna benéfica (KovALESKI; RIBEIRO, 2003; MOURA et al., 2009).

Em virtude dessas exigências e com a mudança do perfil do consumidor, aliadoà conscientização dos riscos da presença de resíduos de agrotóxicos nos frutos, tem-se buscado alternativas ecologicamente apropriadas para o controle de insetos-praga de diversas culturas (CARVAlHo et al., 2000; Moura et al., 2009), dentre elas o controle biológico aplicado e/ou natural.

Nesse sentido, o conhecimento acerca das espécies benéficas presentes em um agroecossistema e que apresentem potencial como agentes de controle biológico de insetos-praga é de suma importância, de modo que se possam adotar medidas dentro de um programa de manejo integrado de pragas que fomentem a conservação e a multiplicação desses organismos. Busca-se com isso reduzir o uso de agrotóxicos no controle de pragas, minimizar o impacto sobre o ambiente e os riscos à saúde humana e atender às exigências dos mercados consumidores.

Assim sendo, objetivou-se com o presente trabalho conhecer os inimigos naturais associados às moscas-das-frutas de ocorrência em pomar urbano de goiabeira, em Fortaleza, Ceará, bem como determinar a flutuação populacional desses insetos benéficos nesse agroecossistema.

\section{MATERIAL E MÉTODOS}

O estudo foi realizado em área experimental da Horta Didática e de Pesquisa da Universidade Federal do Ceará (UFC), localizada no campus do Pici, em Fortaleza, Ceará. No período de $1^{\circ}$ de novembro de 1999 a 27 de outubro de 2000, realizaram-se coletas semanais de frutos maduros provenientes de seis plantas selecionadas aleatoriamente em um pomar de goiabeiras $(6 \mathrm{~m} \times 6 \mathrm{~m})$, de cerca de cinco anos de idade, distribuídas em uma área de cerca de um hectare. Coletaram-se, também, frutos recém-caídos sob as copas das plantas avaliadas, conforme a sua disponibilidade no momento das avaliações. Não se realizou separação de frutos coletados diretamente das plantas daqueles coletados do solo. Foram realizadas 52 avaliações durante o período de estudo.

Os frutos coletados foram acondicionados em sacos de papel com capacidade para $5 \mathrm{~kg}$ e trans- portados para o Laboratório de Entomologia Agrícola da UFC, também localizado no campus do Pici, onde foram quantificados em relação a seu número e sua massa e, posteriormente, transferidos para placas de Petri (20 cm de diâmetro $\times 2,5 \mathrm{~cm}$ de profundidade) contendo vermiculita.

As placas de Petri foram colocadas dentro de gaiolas confeccionadas em acrílico $(40 \mathrm{~cm}$ de altura x $30 \mathrm{~cm}$ de largura $x 30 \mathrm{~cm}$ de comprimento) contendo orifícios de $15 \mathrm{~cm}$ de diâmetro nas laterais e na porta, que foram fechados com tecido fino tipo filó, de modo a permitir a aeração no interior das gaiolas. As gaiolas foram mantidas à temperatura de $29 \pm 3^{\circ} \mathrm{C}$, UR de $75 \pm 10 \%$ e fotofase de $12 \mathrm{~h}$, por um período de 21 dias, de modo a permitir a emergência de inimigos naturais, bem como de larvas de moscas-das-frutas e sua pupação na vermiculita. O número de pupários foi quantificado, sendo eles transferidos para novas placas de Petri contendo vermiculita, até a emergência de adultos (inimigos naturais e moscas-das-frutas). Os adultos coletados foram mortos, quantificados e colocados em frascos de vidro etiquetados $(8 \mathrm{~cm}$ de altura $\times 3 \mathrm{~cm}$ de diâmetro), contendo álcool (70\%), de modo a conservá-los até o momento de sua identificação.

A identificação dos adultos obtidos foi realizada por um especialista, utilizando-se chaves analíticas, no Laboratório de Entomologia Agrícola da UFC. Depois de identificados, os insetos foram quantificados e os dados obtidos utilizados para a confecção de gráficos de flutuação populacional, levando-se em consideração sua densidade populacional.

Dados meteorológicos do período em que a pesquisa foi realizada foram obtidos junto à Estação Meteorológica do Centro de Ciências Agrárias da UFC ( $3^{\circ} 33^{\prime} \mathrm{S}$ e $\left.38^{\circ} 33^{\prime} \mathrm{W}\right)$, localizada a aproximadamente 1,1 km de distância da área experimental, de modo a facilitar o entendimento sobre a flutuação populacional da entomofauna em estudo.

\section{RESULTADOS E DISCUSSÃO}

Ao longo das 52 avaliações realizadas durante o período de condução do estudo foram coletados 43,97 $\mathrm{kg}$ de frutos de goiabeira (531 frutos), de onde foram obtidos 1.335 pupários e 825 adultos de moscas-dasfrutas (Diptera: Tephritidae), sendo 22 espécimes de Anastrepha (Anastrepha sororcula Zucchi e Anastrepha zenildae Zucchi) e 803 espécimes de Ceratitis capitata (Wiedemann) (Fig. 1), além de 75 parasitoides.

Os parasitoides coletados pertencem às famílias Braconidae $(2,7 \%)$, Chalcididae $(2,7 \%)$, Figitidae (Eucoilinae) (74,6\%), Diapriidae (Diapriinae) (17,3\%) e Eulophidae (2,7\%). A ocorrência desses inimigos 
naturais se restringiu aos meses de janeiro, março, julho e agosto de 2000, sendo que as maiores incidências se deram nos meses de janeiro e março. Em janeiro foram coletados 31 adultos de Figitidae e dois adultos de Diapriidae e Eulophidae, respectivamente, enquanto que em março obtiveram-se 25 espécimes de Figitidae e 11 de Diapriidae. Nos meses de julho e agosto de 2000 foram coletados apenas dois espécimes de Chalcididae e Braconidae, respectivamente (Fig. 2).

No que diz respeito aos parasitoides coletados a partir de frutos de goiabeira, acredita-se que muitos destes estejam associados, principalmente, à espécie C. capitata, uma vez que a maior ocorrência de moscas-das-frutas durante a realização da pesquisa foi relativa a essa espécie-praga. No entanto, a associação correta dos parasitoides obtidos com as espécies de moscas-das-frutas de ocorrência em goiabeira no local de estudo não foi possível. Por outro lado, sugere-se que os braconídeos (Hymenoptera: Braconidae) obtidos em agosto de 2000 estejam associados às espécies de Anastrepha coletadas, visto que, segundo ARAUJO;ZUCCHI (2002), esses parasitoides são mais atraídos por voláteis liberados por frutos nativos (como os de goiabeira) atacados por espécies de Anastrepha.

Os resultados obtidos no presente estudo, em relação aos parasitoides coletados a partir de frutos de goiabeira, diferem daqueles de outros autores (MARChiori et al., 2000; ArAujo; ZucCHI, 2002; SouZAFilho et al., 2007; Alvarenga et al., 2009; MarinHo et al., 2009), uma vez que esses autores verificaram maior ocorrência de braconídeos parasitando moscas-das-frutas nessa espécie botânica. Dentre os braconídeos obtidos nos levantamentos realizados pelos autores citados anteriormente, a espécie mais comum foi Doryctobracon areolatus (Szépligeti), parasitoide nativo e considerado o mais importante inimigo natural de moscas-das-frutas no Brasil (ARAujo; ZuCCHI, 2002; UCHÔA-FERNANDES et al., 2003; Carvalho, 2005; Alvarenga et al., 2009; MARINHO et al., 2009). No entanto, parasitoides pertencentes às famílias Diapriidae (Diapriinae), Figitidae e Pteromalidae também têm sido obtidos a partir de larvas e pupas de mocas-das-frutas em goiabeira (MARChIORI et al., 2000; Araujo; ZuCCHI, 2002; SouZa-Filho et al., 2007; Alvarenga et al., 2009; MARINHO et al., 2009).

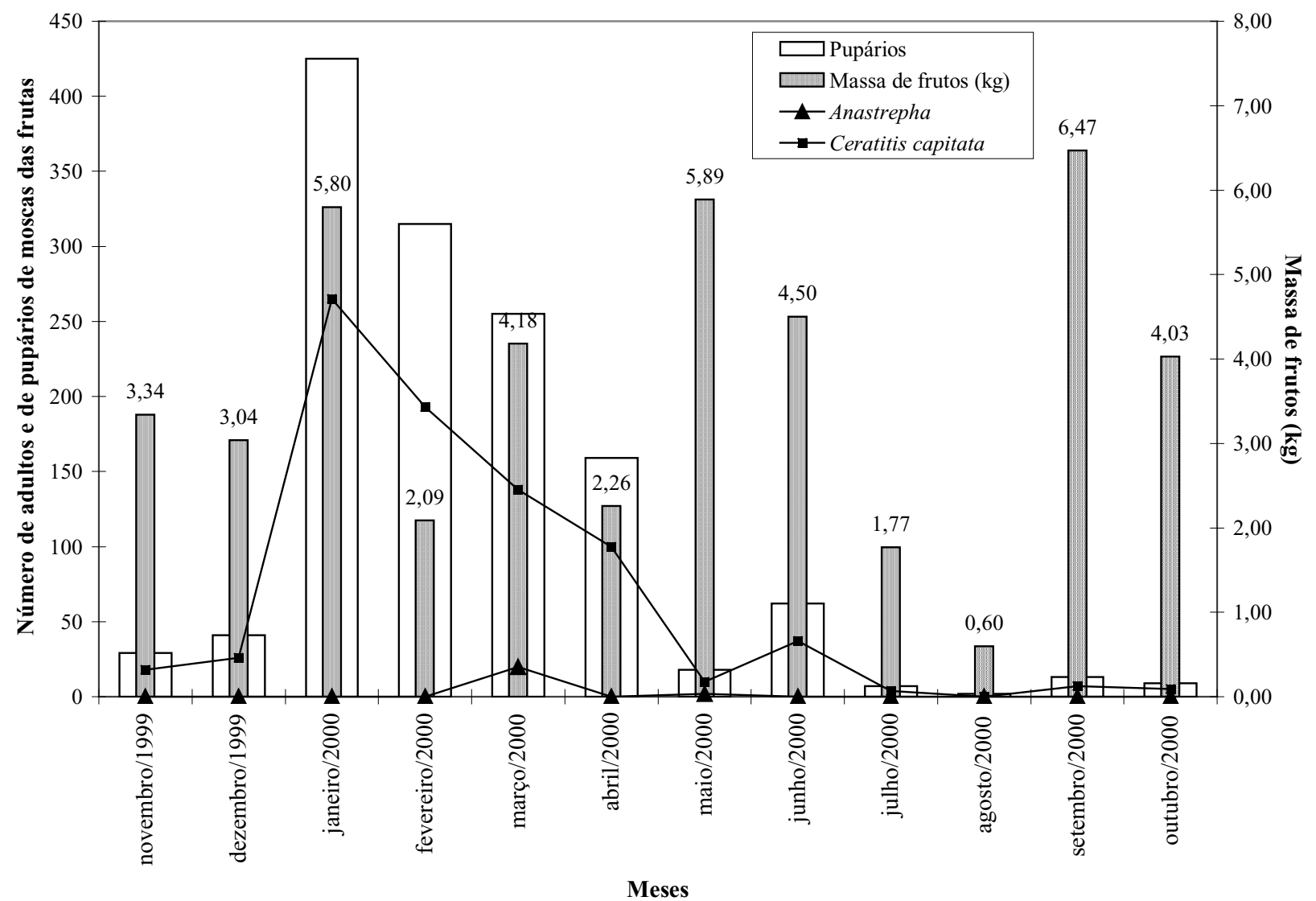

Fig. 1 - Flutuação da coleta de frutos e de adultos de moscas-das-frutas (Diptera: Tephritidae), obtida de frutos de goiabeira coletados em pomar experimental localizado no campus do Pici, em Fortaleza, Ceará, no período de novembro de 1999 a outubro de 2000. 


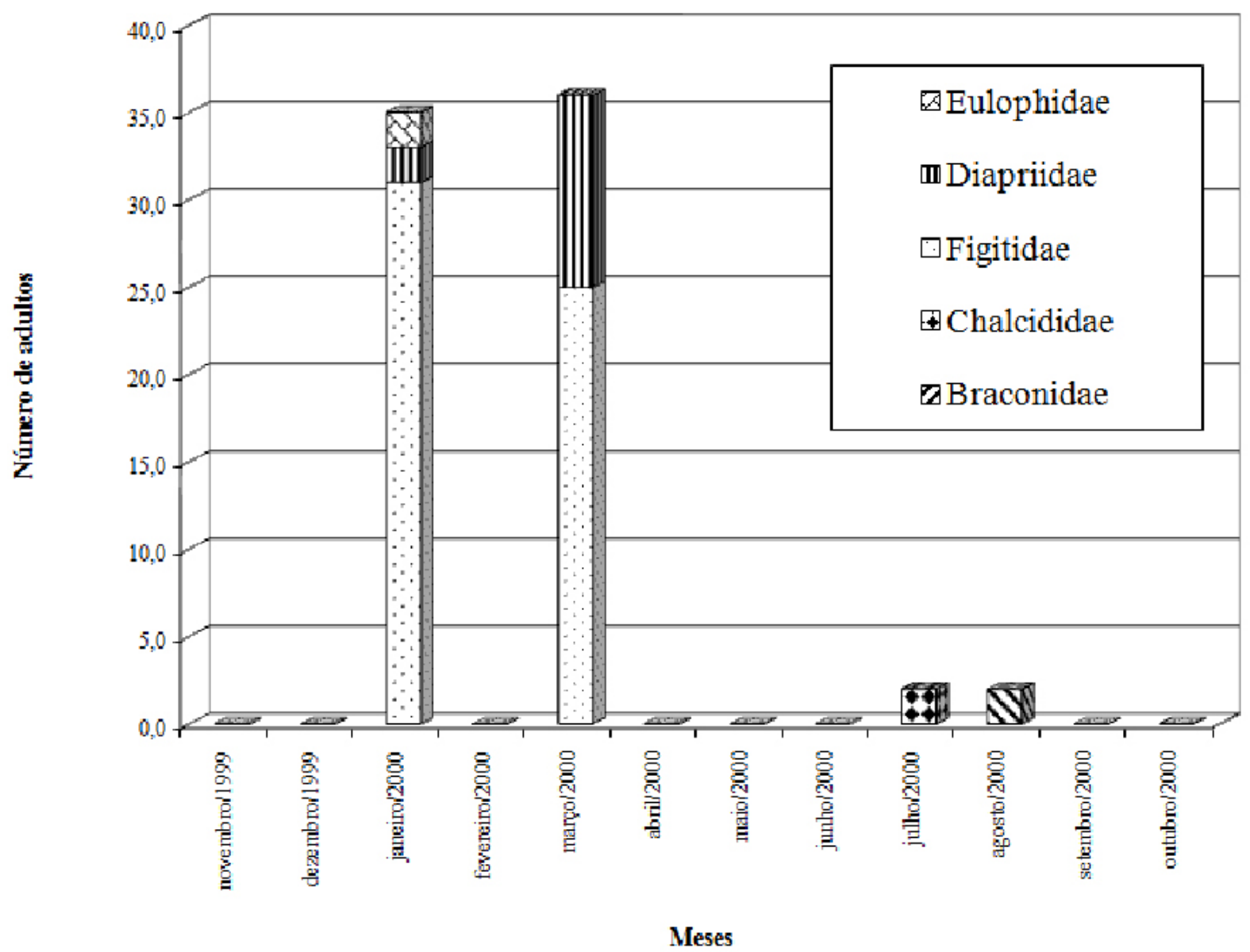

Fig. 2 - Flutuação populacional de inimigos naturais de moscas-das-frutas (Diptera: Tephritidae), das famílias Braconidae, Chalcididae, Figitidae, Diapriidae e Eulophidae, coletados por meio da incubação de frutos de goiabeira em pomar experimental localizado no campus do Pici, em Fortaleza, Ceará, no período de novembro de 1999 a outubro de 2000.

Acredita-se que as diferenças observadas entre o presente estudo eos demais trabalhos citados possam estar associadas à localização da área experimental. Estudos realizados por Alvarenga et al. (2009) evidenciaram que o parasitismo por braconídeos em pomares de goiabeira localizados na área urbana de Janaúba, no Estado de Minas Gerais, foi bastante reduzido, quando em comparação com aqueles pomares localizados em áreas rurais de Jaíba e Nova Porteirinha, também no Estado de Minas Gerais. Além disso, esses autores verificaram que, em áreas urbanas, cerca de $90 \%$ das moscas-das-frutas coletadas pertenciam à espécie $C$. capitata e, em áreas rurais, 93,4\% ao gênero Anastrepha.

Verificou-se que as maiores ocorrências dos inimigos naturais de moscas-das-frutas (janeiro e março de 2000) coincidiram com as maiores incidências desse inseto-praga em goiabeira, notadamente da espécie $C$. capitata, que se deu durante os primeiros quatro meses de 2000. Observou-se, ainda, que durante os meses de janeiro e março do ano de 2000, a presença de frutos de goiabeira foi também elevada, tendo sido coletados $5,8 \mathrm{~kg}$ e 4,18 $\mathrm{kg}$ de goiabas, respectivamente (Figs. 1 e 2).
Acredita-se que as maiores ocorrências de inimigos naturais durante os meses de janeiro e março de 2000, em Fortaleza, Ceará, além de estarem relacionadas à maior disponibilidade de frutos e, consequentemente, de seus hospedeiros (moscasdas-frutas), também foram influenciadas, apesar de indiretamente, pela precipitação pluvial.

Analisando-se a Figura 3, verifica-se que durante os meses de janeiro e março de 2000 a precipitação pluvial total foi de cerca de $230 \mathrm{~mm}$ e $350 \mathrm{~mm}$, respectivamente, o que corresponde a médias diárias de 7,4 mm (janeiro) e 11,3 mm (março). A precipitação observada duranteos meses dejaneiroe março podem ter influenciado, indiretamente, a maior ocorrência de inimigos naturais de moscas-das-frutas durante o período citado, haja vista que esses insetos-praga passam pelo estágio de pupa no solo, sofrendo atuação direta da umidade do solo, a qual depende da precipitação pluvial, fato esse que também foi ressaltado por ARAujo et al. (2008), em estudo realizado em pomar de goiabeira localizado no Município de Russas $\left(5^{\circ} 2^{\prime} \mathrm{Se} 38^{\circ} 2^{\prime} \mathrm{W}\right)$, também no Estado do Ceará.

Assim sendo, a precipitação pluvial atuaria, indiretamente, no desenvolvimento das pupas de 
moscas-das-frutas, na disponibilidade de adultos e, consequentemente, de uma nova geração de larvas presentes nos frutos, de modo a servirem como hospedeiros para os parasitoides presentes no agroecossistema da goiabeira, influenciando a ocorrência e a flutuação populacional desses organismos.

Por outrolado, nos demais meses em que o estudo foi realizado (excetuando-se os primeiros quatro meses do ano de 2000, período de maior ocorrência de moscas-das-frutas), apesar da disponibilidade constante de frutos, a incidência desses insetos-praga no agroecossistema da goiabeira, em Fortaleza, Ceará, foi pequena, coincidindo também com períodos de baixa precipitação pluvial (variando de $0,04 \mathrm{~mm} \mathrm{dia}^{-1}$ a 5,53 $\mathrm{mm} \mathrm{dia}^{-1}$ ), o que pode ter interferido na ocorrência e na flutuação populacional dos parasitoides (Figs. 1, 2 e 3), conforme comentado anteriormente.

Vale ressaltar que a evapotranspiração da cultura (ETc) da goiabeira, estimada para a cultivar Paluma (plantas com cerca de dois anos de idade), na região de Petrolina $\left(9^{\circ} 9^{\prime} \mathrm{S}\right.$ e $\left.40^{\circ} 22^{\prime} \mathrm{W}\right)$, em Pernambuco, foi de 4,53 mm dia ${ }^{-1}$, em média (TeixeIRA et al., 2003). Comparando-se o valor de ETc, obtido por esses autores para a cultura da goiabeira, com os valores de precipitação pluvial média obtidos para os meses de baixa precipitação, observados durante a realização do presenteestudo $\left(0,04 \mathrm{mmdia}^{-1} \mathrm{a} 5,53 \mathrm{~mm} \mathrm{dia}^{-1}\right)$, verificase que a disponibilidade de água no solo na área, em muitos momentos, esteve, provavelmente, abaixo das necessidades hídricas dessa cultura. Em face disso, e baseando-se em estudos realizados por AzEvEDo; PARRA (1989), acredita-se que a umidade do solo pode ter influenciado a ocorrência de moscas-das-frutas e, indiretamente, de seus parasitoides na área de estudo, conformejá comentado. Esses autores verificaram que a falta de umidade do solo (umidade inferior a 5,5\%) podecausarainviabilidade depupas, além de dificultar a emergência de adultos de moscas-das-frutas.

Baseando-se no que foi apresentado no presente trabalho, ressalta-se que estudos que visem o conhecimento sobrea ocorrência e a dinâmica populacional dos inimigos naturais de importantes insetos-praga da fruticultura brasileira e mundial, comoé o caso das moscas-das-frutas (Diptera:Tephritidae), são de suma importância, de modo que se possam adotar medidas efetivas de controle dessas pragas, em consonância com as exigências dos mercados consumidores, notadamente externos, reduzindo-se o impacto negativo do uso de agrotóxicos sobre o ambiente, bem como os riscos à saúde dos trabalhadores e dos consumidores.

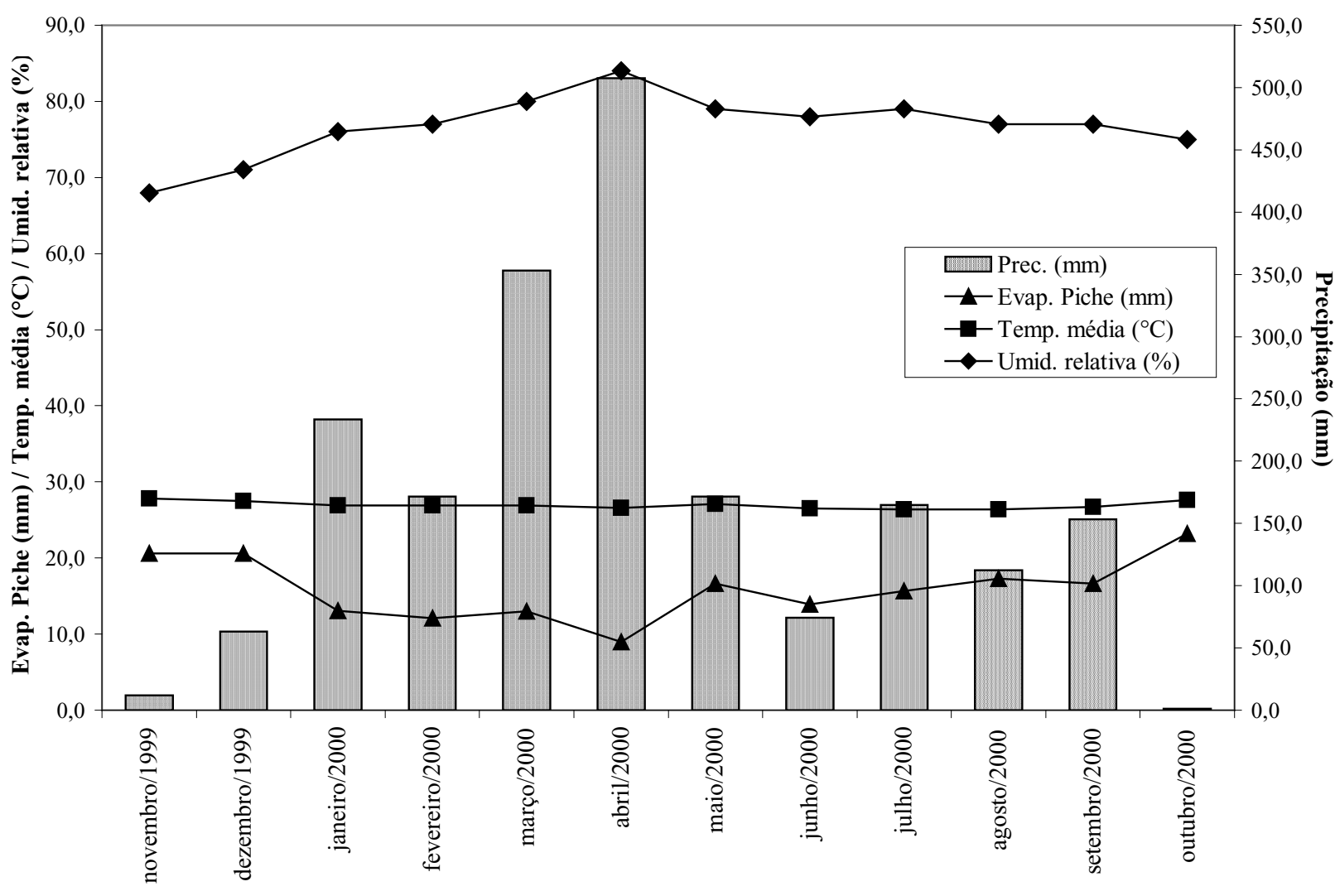

Meses

Fig. 3 - Dados climáticos [precipitação total (mm), temperatura média $\left({ }^{\circ} \mathrm{C}\right)$, umidade relativa $(\%)$ e evaporação total pelo evaporímetro de Piche (mm)] fornecidos pela Estação Meteorológica do Centro de Ciências Agrárias da Universidade Federal do Ceará ( $3^{\circ} 33^{\prime}$ S e 38³3’ W), localizada no campus do Pici, em Fortaleza, Ceará, no período de novembro de 1999 a outubro de 2000 . 


\section{CONCLUSÕES}

Os parasitoides de moscas-das-frutas de ocorrência em goiabeira, em Fortaleza, Ceará, pertencem às famílias Braconidae, Chalcididae, Figitidae (Eucoilinae), Diapriidae (Diapriinae) e Eulophidae;

Parasitoides da família Figitidae (Eucoilinae) são os mais comuns parasitando moscas-das-frutas em Fortaleza, Ceará;

As maiores incidências dos parasitoides na área de estudo ocorreram em janeiro e março de 2000.

\section{AGRADECIMENTOS}

Ao Conselho Nacional de Desenvolvimento Científico e Tecnológico - CNPq, pela concessão de bolsa de estudos ao primeiro autor, e à Dra. Niédja G. G. Gonçalves, pela identificação dos organismos coletados durante a realização da pesquisa.

\section{REFERÊNCIAS}

AGRIANUAL. Anuário da agricultura brasileira. São Paulo: Instituto FNP, 2009. p.325-328.

ALVARENGA, C.D.; MATRANGOLO, C.A.R.; LOPES, G.N.; SILVA, M.A.; LOPES, E.N.; ALVES, D.A.; NASCIMENTO, A.S.; ZUCCHI, R.A. Moscas-dasfrutas (Diptera: Tephritidae) e seus parasitóides em plantas hospedeiras de três municípios do norte do Estado de Minas Gerais. Arquivos do Instituto Biológico, São Paulo, v.76, n.2, p.195-204, 2009. Disponível em: <http://www.biologico.sp. gov.br/docs/arq/v76_2/alvarenga.pdf>. Acesso em: 18 jul. 2009.

ARAUJO, E.L.; ZUCCHI, R.A. Parasitóides (Hymenoptera: Braconidae) de moscas-das-frutas (Diptera: Tephritidae) na Região de Mossoró/Assu, Estado do Rio Grande do Norte. Arquivos do Instituto Biológico, São Paulo, v.69, n.2, p.65-68, 2002.

ARAUJO, E.L.; SILVA, R.K.B.; GUIMARÃES, J.A.; SILVA, J.G.; BITTENCOURT, M.A.L. Levantamento e flutuação populacional de moscas-das-frutas (Diptera: Tephritidae) em goiaba Psidium guajava L., no município de Russas (CE). Revista Caatinga, v.21, n.1, p.138-146, 2008.

AZEVEDO, E.W.V.W.; PARRA, J.R.P. Influência da umidade em dois tipos de solo na emergência de Ceratitis capitata. Pesquisa Agropecuária Brasileira, v.24, n.3, p.321-327, 1989.

CARVALHO, R.S. Avaliação das liberações inoculativas do parasitóide exótico Diachasmimorpha longicaudata (Ashmead) (Hymenoptera: Braconidae) em pomar diversificado em Conceição do Almeida, BA. Neotropical Entomology, v.34, n.5, p.799-805, 2005.

CARVALHO, R.S.; NASCIMENTO, A.S.; MATRANGOLO, W.J.R. Controle biológico. In: MALAVASI, A.; ZUCCHI, R.A. (Ed.). Moscas-das-frutas de importância econômica no Brasil: conhecimento básico e aplicado. Ribeirão Preto: Hollos, 2000. p.113-117.

COSTA, R.I.F.; SILVA, C.G.; MARCHIORI, C.H.; AMARAL, B.B.; POLETTI, M.M.; TORRES, L.C. Parasitismo em Anastrepha sp. (Diptera: Tephritidae) por Aganaspis pelleranoi (Brèthes, 1924) e Dicerataspis sp. (Hymenoptera: Figitidae: Eucoilinae). Ciência e Agrotecnologia, v.31, n.3, p.720-723, 2007.

KOVALESKI, A.; RIBEIRO, L.G. Manejo de pragas na produção integrada de maçã. In: PROTAS, J.F.S.; SANHUEZA, R.M.V. (Ed.). Produção integrada de frutas: o caso da maçã no Brasil. Bento Gonçalves: Embrapa Uva e Vinho, 2003. p.61-68.

MALAVASI, A. Áreas-livres ou de baixa prevalência. In: MALAVASI, A.; ZUCCHI, R.A. (Ed.). Moscas-das-frutas de importância econômica no Brasil: conhecimento básico e aplicado. Ribeirão Preto: Hollos, 2000. p.175-181.

MARCHIORI, C.H.; OLIVEIRA, A.M.S.; MARTINS, F.F.; BOSSI, F.S.; OLIVEIRA, A.T. Espécies de moscas-das-frutas (Diptera: Tephritidae) e seus parasitóides em Itumbiara-GO. Pesquisa Agropecuária Tropical, v.30, n.2, p.73-76, 2000.

MARINHO, C.F.; SOUZA-FILHO, M.F.; RAGA, A.; ZUCCHI, R.A. Parasitóides (Hymenoptera:

Braconidae) de moscas-das-frutas (Diptera: Tephritidae) no Estado de São Paulo: plantas associadas e parasitismo. Neotropical Entomology, v.38, n.3, p.321-326, 2009.

MOURA, A.P.; CARVALHO, G.A.; MOSCARDINI, V.F.; MARQUES, M.C.; SOUZA, J.R. Toxicidade de pesticidas recomendados na produção integrada de maçã (PIM) a populações de Chrysoperla externa (Hagen) (Neuroptera: Chrysopidae). Neotropical Entomology, v.38, n.3, p.395-404, 2009.

PEREIRA, F.M. Cultura da goiabeira. Jaboticabal: FUNEP, 1995. 47p.

SOUZA-FILHO, Z.A.; ARAUJO, E.L.; GUIMARÃES, J.A.; SILVA, J.G. Endemic parasitoids associated with Anastrepha spp. (Diptera: Tephritidae) infesting guava (Psidium guajava) in Southern Bahia, Brazil. Florida Entomologist, v.90, n.4, p.783-785, 2007.

TEIXEIRA, A.H.C.; BASSOI, L.H.; REIS, V.C.S.; SILVA, T.G.F.; FERREIRA, M.N.L.; MAIA, J.L.T. Estimativa do consumo hídrico da goiabeira, utilizando estações agrometeorológicas automática e convencional. Revista Brasileira de Fruticultura, v.25, n.3, p.457-460, 2003. 
UCHÔA-FERNANDES, M.A.; MOLINA, R.M.S.; OLIVEIRA, I.; ZUCCHI, R.A.; CANAL DAZA, N.A.; DÍAZ, N.B. Larval parasitoids (Hymenoptera) of frugivorous flies (Diptera, Tephritoidea) reared from fruits of the cerrado of the State of Mato Grosso do Sul, Brazil.
Revista Brasileira de Entomologia, v.47, n.2, p.181-186, 2003.

Recebido em 27/10/09

Aceito em 17/1/11 